\title{
An interatomic pair potential with tunable intrinsic ductility
}

\author{
V. P. Rajan ${ }^{1}$, D. H. Warner ${ }^{2}$ and W. A. Curtin ${ }^{1}$ \\ ${ }^{1}$ Institute of Mechanical Engineering, École Polytechnique Fédérale de Lausanne, \\ Lausanne, CH-1015, Switzerland \\ ${ }^{2}$ School of Civil and Environmental Engineering, Cornell University, Ithaca, NY \\ 14853, USA
}

\begin{abstract}
A family of interatomic potentials is constructed for which the intrinsic ductility can be tuned systematically. Specifically, the elastic constants and critical energy release rate for Griffith cleavage, $G_{I c}$, are held constant, while the critical energy release rate for dislocation emission, $G_{I e}$, can be varied. This behavior is achieved by modifying a standard near-neighbor pair potential; the new potential is applicable to either 2D (hexagonal lattice) or 3D (FCC/HCP). Analytical expressions are provided for $G_{I e}$ and $G_{I c}$, enabling a potential with a desired intrinsic ductility to be easily developed. Direct atomistic simulations are used to demonstrate that the new potentials control the intrinsic material ductility, i.e. crack tip dislocation emission versus brittle cleavage, under quasi-static loading. For the 2D potential, the mode I crack tip behavior can be tuned from brittle to ductile; for the 3D potential, such tuning is only possible for certain crack orientations. More generally, the new potentials are expected to be useful in a wide range of physical problems in which behavior is controlled by the ability of the material to nucleate dislocations, including problems involving crack tips, grain boundaries, contact and friction, and bi-material interfaces. ${ }^{1}$
\end{abstract}

\section{Introduction}

Atomistic simulations provide insights into material deformation and fracture that can be inaccessible to coarser-scale methods [1]. Such simulations rely on interatomic poten-

\footnotetext{
${ }^{1}$ This is an author-created, un-copyedited version of an article accepted for publication/published in Modelling and Simulation in Materials Science. IOP Publishing Ltd. is not responsible for any errors or omissions in this version of the manuscript or any version derived from it. The version of record is available online at dx.doi.org/10.1088/0965-0393/24/2/025005
} 
tials to characterize the forces between atoms and the energies of atomic configurations. Material-specific potentials are naturally required for investigations of material-specific deformation mechanisms (e.g., twinning in FCC metals [2]). However, quantitatively reliable potentials are not available for many materials, especially non-FCC crystals. More importantly, many mechanics phenomena are quite general, being observable across different materials and even different classes of materials. For instance, in dynamic fracture, crack tip instabilities are simply the product of brittle, short-range interactions between atoms, and, as such, can be seen in materials as diverse as (brittle) polymers, ceramics, and glasses [3,4]. Model interatomic potentials enable the general principles of material deformation and fracture to be elucidated without confining attention to a particular material system. Buehler et al. [5], for instance, used an artificial pair potential to investigate the limiting crack speed for dynamic fracture in a brittle material. This family of potentials allowed for tunable "hyperelasticity", which was demonstrated to correlate with the limiting speed. In the present work, we adopt a similar approach in spirit and construct a family of potentials for which the intrinsic ductility can be tuned systematically.

The concept of intrinsic ductility, made quantitative by Rice and Thomson [6] and Rice [7], characterizes the competition between two different modes of deformation in a material with a crack: Griffith cleavage (the brittle mode) and dislocation emission from the crack tip (the ductile mode). Each mode requires a characteristic energy to be activated. For (Mode I) Griffith cleavage, this quantity is termed $G_{I c}$ and equals twice the surface energy of the cleavage plane,

$$
G_{I c}=2 \gamma_{s}
$$

For Mode I dislocation emission, this quantity is termed $G_{I e}$ and is related to the unstable stacking fault energy $\gamma_{u s}$ through the relationship [7]:

$$
G_{I e}=\frac{8 \gamma_{u s}}{(1+\cos \theta) \sin ^{2} \theta}
$$

for emission of dislocations along a slip plane oriented at an angle $\theta$ to the crack plane, and with the line direction parallel to the crack front. For quasi-static loading, if $G_{I c}<G_{I e}$, the material is expected to be intrinsically brittle: that is, cleavage should occur before dislocation emission and attendant crack blunting. Conversely, if $G_{I e}<G_{I c}$, then the material should be ductile: that is, the crack will emit dislocations and blunt rather than cleave. Hence, the ratio $G_{I c} / G_{I e}$ is a measure of the intrinsic ductility of a given material.

Here, we develop a family of potentials for which $G_{I e}$ can be tuned over a wide range independently of $G_{I c}$, with near-equilibrium properties of the material, such as the elastic constants, remaining constant. Thus, the material can be tuned from brittle to ductile. For instance, since the elastic constants are held fixed, these potentials can be used to study bi-material systems in which only the ductility of the materials is varied, thus avoiding 
elastic mismatch stresses, wave reflection in dynamics simulations, and other complications that might obscure the underlying role of the ductility. We achieve this behavior by using a near-neighbor pair potential for which (i) the surface energy and elastic constants are determined solely by the energy-distance relationship near equilibrium: that is, near the energy minimum of the potential and (ii) the unstable stacking fault energy is determined solely by the large-separation "tail" of the potential. The intrinsic ductility of a potential can thus be tuned by altering the tail of the potential while leaving the behavior near the minimum unchanged. Holian and Ravelo [8] explored a similar idea but their potentials were constructed in an ad hoc manner and without any connection to the Rice [7] analysis of intrinsic ductility.

The paper is organized as follows. We first describe the functional form of our new family of potentials, applicable both to 2D (hexagonal lattice) and 3D (FCC/HCP) crystals. Certain parameters vary with dimensionality, however. The analytical form of the potential is exceedingly simple, allowing the elastic properties and characteristic energies $\left(\gamma_{s}, \gamma_{u s}\right)$ to be calculated analytically. We construct potentials spanning a range of $\gamma_{u s}$ : i.e., varying $G_{I e}$ and keeping $G_{I c}$ fixed. Finally, as a case study, we validate the behavior of the potentials by conducting quasi-static fracture tests using direct atomistic simulations. (The study of dynamic fracture is presented in a forthcoming publication [9].) In 2D, the crack tip can indeed be tuned from brittle to ductile. In 3D (FCC), such tuning is also possible, but only for specific crack orientations. We note, however, that the new potentials are expected to be useful in a broad range of simulations where the intrinsic ductility governs the competition between modes of deformation.

\section{Development of the family of potentials}

We develop a near-neighbor potential characterized by the energy function $\varphi(r)$, where $r$ is the distance between atom centers. We express all quantities in non-dimensional form. All energies are normalized by the depth of the energy well $\epsilon$, all lengths by the equilibrium distance $r_{0}$, and all masses by the atomic mass $m$. Thus, in normalized form, $\varphi(1)=-1$. In this system of units, the associated unit of time is $r_{0} \sqrt{m / \epsilon}$, of velocity is $\sqrt{\epsilon / m}$, of elastic moduli is $\epsilon / r_{0}^{3}$, and of energy release rate is $\epsilon / r_{0}^{2}$.

As stated previously, the intrinsic ductility of the potential can be tuned by altering the tail of $\varphi(r)$ while leaving the energy well unchanged. In light of this, we modify a standard 
Morse potential by using a piecewise-continuous "tail," with the specific form

$$
\varphi(r)= \begin{cases}(1-\exp [-\alpha(r-1)])^{2}-1 & r \leq r_{1} \\ A_{1} r^{3}+B_{1} r^{2}+C_{1} r+D_{1} & r_{1}<r \leq r_{2} \\ A_{2} r^{3}+B_{2} r^{2}+C_{2} r+D_{2} & r_{2}<r \leq r_{3} \\ 0 & r_{3}<r\end{cases}
$$

where the distances $\left(r_{1}, r_{2}, r_{3}\right)$, and the cubic spline coefficients $\left(A_{1}, B_{1}, \ldots D_{2}\right)$ are determined by various considerations, as follows.

First, since $\varphi(r)$ describes a near-neighbor pair potential, we impose $r_{3}<r_{n n n}$, where $r_{n n n}$ is the next-nearest-neighbor distance in the equilibrium structure. In $2 \mathrm{D}, r_{n n n}=\sqrt{3}$; in $3 \mathrm{D}$ FCC or ideal HCP, $r_{n n n}=\sqrt{2}$. Second, it is desirable that the behavior of each potential be identical up to a few percent deformation so that no elastic mismatch arises at finite strains in bi-material simulations. Here, we select $r_{1}=1.05$ so that the potentials are matched in tension to approximately $5 \%$ strain, and we select $r_{3}=r_{n n n}-0.02$ so that the potentials are matched in compression to roughly $2 \%$. The actual degree of matching can be altered as desired, however, and is not an intrinsic feature of the new class of potentials.

The elastic properties are set by the parameter $\alpha$ of the Morse potential, where $\alpha^{2}$ is proportional to the curvature $\mathrm{d}^{2} \varphi / \mathrm{d} r^{2}$ of the potential around the minimum energy. Again, this value can be adjusted within the potential family; however, we have obtained good results using $\alpha=7 / 2^{(5 / 6)} \approx 3.93$ in $2 \mathrm{D}$ and $\alpha=13 / 2^{(5 / 6)} \approx 7.30$ in $3 \mathrm{D}$. Substantially larger values of $\alpha$ limit the attainable range of $\gamma_{u s}$, whereas substantially smaller values cause higher-order derivatives of $\varphi(r)$ for the brittle potentials to be highly discontinuous and non-monotonic. Using standard expressions for zero-temperature elastic constants, we find that the 2D hexagonal lattice is elastically isotropic with Poisson's ratio $v=0.25$ and shear modulus $\mu=\sqrt{3} \alpha^{2} / 2$. For the 3D FCC lattice with cubic symmetry, the nonzero components of the stiffness tensor in Voigt notation $C_{i j}$ relative to the cube axes are $C_{11}=2^{3 / 2} \alpha^{2}$ and $C_{12}=C_{66}=C_{11} / 2$. Using the selected values of $\alpha, \mu \approx 13.4$ in $2 \mathrm{D}$ and $C_{11} \approx 150.6$ and $C_{12}=C_{66} \approx 75.3$ in 3D. The surface energy in $2 \mathrm{D}$ is $\gamma_{s}=1$ and the surface energy for the $\{111\}$ plane of FCC and the basal plane of HCP is $\gamma_{s}=\sqrt{3}$, so the Griffith fracture energies are

$$
G_{I c}= \begin{cases}2 & (2 \mathrm{D}) \\ 2 \sqrt{3} & (3 \mathrm{D}, \text { close-packed plane })\end{cases}
$$

Thus, for a single choice of $\alpha$, the elastic moduli, surface energies, and fracture energy are fixed for all potentials.

Seven of the remaining eight unknown spline coefficients $\left(A_{1}, B_{1}\right.$, etc. $)$ are determined using continuity conditions. Continuity in the energy $\varphi(r)$ and the force $\varphi^{\prime}(r)$ are imposed 
at $r_{1}, r_{2}$, and $r_{3}$, and continuity in the curvature $\varphi^{\prime \prime}(r)$ is imposed at $r_{2}$. We select $r_{3}$ to be the largest possible value such that the second cubic spline is always less than zero (attractive) at long distances, recalling that $r_{3}$ is bounded below by $r_{2}$ and above by $r_{n n n}-0.02$. These conditions do not affect the elastic moduli, surface energies, or fracture energies.

The final adjustable constant is chosen to achieve a desired unstable stacking fault energy $\gamma_{u s}$. The unstable stacking fault configuration in a 2D hexagonal lattice is shown in Figure 1(b). We assume that atoms are permitted to relax in the direction perpendicular to the stacking fault: that is, our estimate of $\gamma_{u s}$ corresponds to the relaxed, as opposed to the unrelaxed, configuration [10,11]. As shown in Figure 1, the system opts for a larger lateral distance between planes in the relaxed configuration than in the unrelaxed configuration, in order to minimize the energetically costly repulsive interaction. We assume that $r_{0}^{\prime}$, the distance between nearest-neighbors in the relaxed configuration, equals the equilibrium distance: $r_{0}^{\prime} \approx r_{0}=1$. Denoting the distance between second-nearest neighbors as $r_{2}$, we find from simple geometry that $r_{2}=\sqrt{2}$ (in 2D). The energy of the relaxed, unstable configuration in $2 \mathrm{D}$, per unit area, then becomes

$$
E_{u s} \approx \varphi(1)+2 \varphi\left(r_{2}\right)=2 \varphi\left(r_{2}\right)-1
$$

The unstable stacking fault energy is the difference between this unstable energy and the equilibrium energy, per unit area, where $E_{e q}=2 \varphi(1)=-2$, leading to

$$
\gamma_{u s}=E_{u s}-E_{e q} \approx 2 \varphi\left(r_{2}\right)+1
$$

A similar analysis for slip along close-packed planes in 3D (FCC or HCP), again assuming $r_{0}^{\prime} \approx 1$ in the relaxed configuration, gives

$$
\gamma_{u s} \approx \frac{4 \varphi\left(r_{2}\right)+2}{\sqrt{3}}
$$

for a partial dislocation, where now $r_{2}=\sqrt{3 / 2}$ in 3D FCC and ideal HCP crystals. The unstable stacking fault energy can thus be tuned independently of the surface energy by modifying the value of $\varphi\left(r_{2}\right)$, the energy between second-nearest neighbors in the unstable, relaxed configuration.

The above analytical result for $\gamma_{u s}$ is not precise since $r_{0}^{\prime}$ does not exactly equal unity in the minimum energy (relaxed) configuration; as a consequence, the energy of the relaxed configuration is (slightly) overestimated by the analytical method. Direct molecular statics simulations thus reveal that $\gamma_{u s}$ as computed by Equations 6 and 7 is consistently too large. However, remarkably, adding a constant correction of -0.088 in $2 \mathrm{D}$ and -0.065 in 3D in Equations 6 and 7, respectively, enables $\gamma_{u s}$ to be estimated to within a few percent for essentially all potentials within this family. With the above conditions, we therefore have a complete, fully-analytical recipe for producing an interatomic potential with the 
desired $G_{I c}$ and $G_{I e}$. In prescribing $G_{I e}$, the slip system must be defined. In 2D, slip occurs via full dislocations oriented at $\pm 60^{\circ}$ to the crack plane. In $3 \mathrm{D}$ (FCC), slip occurs via partial dislocations of type $\sqrt{2} / 6\langle 121\rangle$, whose orientation $\theta$ with respect to the crack plane depends on the orientation of the crack plane and crack front.

\section{Performance of the potentials in quasi-static fracture}

We have constructed several specific potentials for both the 2D and 3D cases, for which the unstable stacking fault energy varies over as wide a range as possible within the imposed constraints. In other words, the potentials range from as brittle to as ductile as possible. The energy and force versus interatomic separation are shown in Figures 2 and 3, and the parameters used to obtain these potentials are summarized in Tables 1 and 2. Although the spline constants can be calculated from these parameters and the continuity conditions enumerated above, we also tabulate these constants in the Tables 3 and 4 for convenience. ${ }^{2}$ In Figures 2 and 3, the energy and force for different potentials are labelled according to the intrinsic ductility, with smaller values of $G_{I e} / G_{I c}$ indicating a more ductile potential. Finally, Tables 1 and 2 also show the analytical predictions for the ratio $G_{I e} / G_{I c}$, recalling that $G_{I c}$ is the same for all potentials. ${ }^{3}$

Generalized stacking fault energy curves for the 2D and 3D potentials are depicted in Figure 4. Again, the curves vary monotonically between the least intrinsically ductile potential and the most. The unstable stacking fault energy is always attained at half the slip displacement, either for a full dislocation (in 2D), or for a partial dislocation (in 3D). In $3 \mathrm{D}$, the energy of the slipped or faulted configuration is the same as that of the original configuration, since the nearest-neighbor potential is unable to distinguish between FCC and HCP; in other words, the stable stacking fault energy equals 0.

We have also performed direct quasi-static molecular simulations using standard procedures for "K/G-tests" $[15,16]$. Specifically, a square domain is centered on a crack tip and the displacements of atoms at the outer boundary of the domain are fixed according to the $K_{I}$ displacement field for an elastic material. In 2D, the isotropic displacement field is used; in 3D, the anisotropic displacement field of Sih et al. [17] is used, along with periodic boundary conditions parallel to the crack front (in the out-of-plane direction). After finding a stable crack configuration at a load below $G_{I e}$ and $G_{I c}$ (if possible), the load $(K / G)$

\footnotetext{
${ }^{2}$ In the supplementary material, we have also provided Python code for generating the various potentials, with an example shown in an IPython notebook [12].

${ }^{3}$ Some of the aforementioned results were generated using a suite of Python tools, including NumPy (Version 1.8.1, [13]), Matplotlib (Version 1.3.1, [14]), and IPython (Version 0.12, [12]).
} 
is increased slightly, and the system is re-equilibrated to find the new crack configuration. At some critical load, crack tip deformation corresponding to either crack advance $\left(G_{I c}\right)$ or dislocation emission $\left(G_{I e}\right)$ occurs. In $2 \mathrm{D}$, the load step is approximately $0.015 K_{I c}$ and the simulation box size is $500 \times 500$; in $3 \mathrm{D}$, the load step is $0.005 K_{I c}$ and the box size is roughly $300 \times 300 \times 2$, depending on the crystallographic orientation of the crack. The simulations are run using the Large-scale Atomic/Molecular Massively Parallel Simulator (LAMMPS) (Version June 28, 2014, Plimpton [18]), and the results are visualized using the Open Visualization Tool (OVITO) (Version 2.8.2, Stukowski [19]). Note that the K/G-test is only able to measure the critical value of $G$ for the most favorable (lowest $G$ ) process; that is, the crack tip event-cleavage or dislocation emission-corresponds to the process with the lowest energy barrier. In 3D, two crack systems were examined. In the first, the crack plane is (111) and the crack front is [110], so that partial dislocations are emitted at $71^{\circ}$ to the crack plane. In the second, the crack plane is (110) and the crack front is [110], so that partial dislocations are emitted at $35^{\circ}$ to the crack plane. We select these orientations because they correspond to the most ductile orientations in FCC, since the slip plane contains the line of the crack. These orientations govern fracture in FCC; if a crack is brittle even in the most ductile orientations, then the material must be fundamentally brittle. Other orientations, where the slip plane intersects the crack front obliquely (for instance, where the crack plane is (110) and the crack front is [001]), are thus generally much less important. A further problem with such orientations is that there is no analytic model for dislocation emission, and, as a practical matter, the use of periodic boundary conditions in the out-of-plane direction, combined with the small dimension of the box in this direction, inhibits dislocation emission, causing the crack tip behavior to appear artificially brittle [20].

Table 1 shows the 2D simulation results for the value of $G_{I c}$ or $G_{I e}$, normalized by the analytical (exact) value for $G_{I c}$ which, again, is the same for all potentials. The simulation results generally compare very well with the analytical predictions in 2D. The Rice predictions for $G_{I e}$ for the ductile potentials are 5-10\% lower than the direct simulated values. The measured values of $G_{I c}$ for the brittle potentials are roughly $10-20 \%$ larger than the Griffith energy; the latter discrepancy arises because of lattice trapping of the crack, which increases with decreasing range of the potential [21]. That is, denoting the value of $G_{I c}$ inclusive of trapping as $G_{I c}^{+}$(whereas $G_{I c}=2 \gamma_{s}$ ), and assuming that the value of $G_{I c}$ measured in simulation is approximately $G_{I c}^{+}$, we find that $G_{I c}^{+} / G_{I c} \approx 1.1-1.2$.

Table 2 shows the 3D simulation results for the value of $G_{I c}$ or $G_{I e}$, normalized by the Griffith energy, $G_{I c}=2 \gamma_{s}$. In 3D, agreement between simulation and prediction is less good, but still acceptable. For the (111)[110] crack orientation, only dislocation emission from the crack tip is observed. The Rice predictions are consistently too low, by $10-40 \%$, with the difference decreasing considerably as the potential becomes more brittle. Some of the 
discrepancy may arise because the relaxed configuration for which $\gamma_{u s}$ was calculated is not truly representative of a dislocation Peierls zone; instead, the appropriate value of $\gamma_{u s}$ lies somewhere between that in the relaxed and unrelaxed configurations [11]. Incorporating this effect would revise the Rice estimate of $G_{I e}$ upwards, into better agreement with the simulation data. Another important effect for the most ductile potentials is that measuring a proper $G_{I e}$ is difficult since the crack heals before emitting, and thus $G_{I e}$ for an initial stable crack is not being measured. For the most ductile potential, this effect is so severe that it is necessary to simulate a blunted crack, created by deleting a row of atoms. The nonzero crack tip radius increases the measured $G_{I e}$ relative to the prediction for the sharp crack, however $[15,22]$. For the most brittle potentials, the fact that cleavage is not observed is initially surprising, considering that $G_{I c}<G_{I e}$. We speculate that lattice trapping elevates $G_{I c}$ above $G_{I e}$ for these potentials: that is, $G_{I c}<G_{I e}<G_{I c}^{+}$. Lattice trapping is especially pronounced in the 3D potential because the second-nearest-neighbor distance, which governs the range of the potential, is much smaller in 3D than in 2D. Significant trapping is indeed observed in the other crack system, (110) [110]. Turning now to this orientation, emission is observed for the most ductile potential, at a value again significantly larger than the Rice prediction. Cleavage is observed for the other potentials, since $G_{I e}$ in this orientation is generally even larger than $G_{I c}^{+}$. The simulation results imply that $G_{I c}^{+} / G_{I c} \approx 1.2-1.6$ : significantly larger than that in 2D. For the most brittle potential, trapping is so severe that the crack cleaves by kinking onto a (111) plane instead of by propagating on the original (110) plane.

The extensive trapping in 3D limits the utility of the potential family, since the critical $G$ for emission cannot be varied truly independently of that for cleavage. Indeed, accurate analytical estimates cannot be given for $G_{I c^{\prime}}^{+}$, although simple models for trapping, such as that of Curtin [21], may provide some guidance. However, even with lattice trapping, tuning the crack behavior from brittle to ductile is possible for certain crack orientations in 3D, such as (110) [110] in FCC and $(\overline{1010})[1 \overline{2} 10]$ in HCP (for the latter, see results in Rajan and Curtin [9]). Thus, at least for these orientations, the potential remains useful for systematic studies of the role of intrinsic ductility in fracture. We also note that lattice trapping is irrelevant to simulations of dynamic fracture; cracks can be made to run dynamically below $G_{I c}^{+}$, but not below $G_{I c}=2 \gamma_{s}$ [3]. Therefore, many of the difficulties associated with the 3D potential family are ameliorated in dynamic applications. Finally, we note that $\gamma_{u s}$ is a more fundamental quantity than $G_{I e}$, characterizing not only the barrier to mode I dislocation nucleation at a sharp crack, but also that for mixed mode loading and nucleation from blunted cracks, surface steps or corners [23], etc. Because the 3D potential enables $\gamma_{u s}$ to be varied over a wide range, independent of the elastic constants, it may therefore also prove useful in simulations where dislocation nucleation governs plasticity, but such nucleation does not originate from a sharp crack tip (e.g., indentation or friction). 


\section{Summary}

We have developed a new family of near-neighbor pair potentials for which the intrinsic ductility can be tuned systematically. Specifically, the energy barrier for dislocation emission from the crack tip, $G_{I e}$, can be changed while leaving the Griffith cleavage energy, $G_{I c}$, and the elastic constants unaffected. The fracture properties of the potentials in mode I were assessed using direct molecular simulations. In the 2D system, both brittle and ductile crack tip behavior can be attained. Lattice trapping of the crack is minimal, and the Rice [7] estimate of $G_{I e}$ is very accurate, so that the agreement between predicted and measured values of $G_{I c}$ and $G_{I e}$ is excellent. In the 3D FCC system, this agreement is poorer, primarily because the shorter cutoff for the potential causes greater lattice trapping, elevating $G_{I c}^{+}$significantly above the Griffith energy. Therefore, in certain orientations in 3D, such as (111)[110], only ductile crack tip behavior is observed. However, in other orientations, such as (110) [110] in FCC and (1010)[1210] in HCP, the direct atomistic tests reveal both brittle and ductile crack tip behavior. This orientation dependence restricts the applicability of the 3D potential family, although, notably, the effects of lattice trapping disappear in dynamic fracture. Therefore, while in principle a potential with a desired ductility can be constructed using our analytical estimates for $G_{I c}$ and $G_{I e}$, in practice the parameters of the potential must be selected carefully, at least in $3 \mathrm{D}$, to account for the effects of trapping.

The new family of potentials is expected to be broadly applicable to molecular simulations that aim to explore the general effects of material ductility on mechanical behavior (as opposed to material-specific slip systems, deformation mechanisms, etc.). As an example, in forthcoming work we use these potentials to investigate the behavior of dynamic cracks running from a brittle material into a ductile material [9]. In this case, these potentials enable systematic exploration of the effects of material fracture properties on dynamic crack tip response without complications arising from elastic or crystallographic mismatch at the bi-material interface. The potential may also find use in many classes of problems where there are competing modes of fracture and ductility, including problems involving dislocation nucleation and plasticity where the unstable stacking fault energy plays an important role.

\section{Acknowledgements}

VPR and WAC gratefully acknowledge funding of this work through a European Research Council Advanced Grant, "Predictive Computational Metallurgy", ERC Grant agree- 
ment No. 339081-PreCoMet. DHW acknowledges funding support from Paul Hess at ONR (Grant No. N000141010323) and Ali Sayir at AFOSR (FA95501110273).

\section{References}

[1] J. Li, A. H. W. Ngan, and P. Gumbsch. Atomistic modeling of mechanical behavior. Acta Materialia, 51(19):5711-5742, 2003.

[2] D. H. Warner and W. A. Curtin. Origins and implications of temperature-dependent activation energy barriers for dislocation nucleation in face-centered cubic metals. Acta Materialia, 57(14):4267-4277, 2009.

[3] M. Marder. Effects of atoms on brittle fracture. International Journal of Fracture, 130 (2):517-555, 2004.

[4] K. Ravi-Chandar. Dynamic fracture of nominally brittle materials. International Journal of Fracture, 90(1-2):83-102, 1998.

[5] M. J. Buehler, F. F. Abraham, and H. Gao. Hyperelasticity governs dynamic fracture at a critical length scale. Nature, 426(6963):141-146, 2003.

[6] J. R. Rice and R. Thomson. Ductile versus brittle behaviour of crystals. Philosophical Magazine, 29(1):73-97, 1974.

[7] J. R. Rice. Dislocation nucleation from a crack tip: an analysis based on the Peierls concept. Journal of the Mechanics and Physics of Solids, 40(2):239-271, 1992.

[8] B. L. Holian and R. Ravelo. Fracture simulations using large-scale molecular dynamics. Physical Review B, 51(17):11275, 1995.

[9] V. P. Rajan and W. A. Curtin. Crack tip blunting and cleavage under dynamic conditions. Submitted, Journal of the Mechanics and Physics of Solids.

[10] J. A. Zimmerman, H. Gao, and F. F. Abraham. Generalized stacking fault energies for embedded atom FCC metals. Modelling and Simulation in Materials Science and Engineering, 8(2):103, 2000.

[11] Y. Sun, G. E. Beltz, and J. R. Rice. Estimates from atomic models of tension-shear coupling in dislocation nucleation from a crack tip. Materials Science and Engineering: A, 170(1):67-85, 1993.

[12] F. Pérez and B. E. Granger. IPython: a system for interactive scientific computing. Computing in Science and Engineering, 9(3):21-29, 2007. 
[13] S. Van Der Walt, S. C. Colbert, and G. Varoquaux. The NumPy array: a structure for efficient numerical computation. Computing in Science \& Engineering, 13(2):22-30, 2011.

[14] J. D. Hunter. Matplotlib: A 2D graphics environment. Computing in Science and Engineering, 9(3):90-95, 2007.

[15] P. Gumbsch. An atomistic study of brittle fracture: Toward explicit failure criteria from atomistic modeling. Journal of Materials Research, 10(11):2897-2907, 1995.

[16] Z. Wu and W. A. Curtin. Brittle and ductile crack-tip behavior in magnesium. Acta Materialia, 88:1-12, 2015.

[17] G. C. Sih, P. C. Paris, and G. R. Irwin. On cracks in rectilinearly anisotropic bodies. International Journal of Fracture Mechanics, 1(3):189-203, 1965.

[18] S. Plimpton. Fast parallel algorithms for short-range molecular dynamics. Journal of Computational Physics, 117(1):1-19, 1995.

[19] A. Stukowski. Visualization and analysis of atomistic simulation data with OVITOthe open visualization tool. Modelling and Simulation in Materials Science and Engineering, 18(1):015012, 2010.

[20] V. Shastry and D. Farkas. Molecular statics simulation of fracture in $\alpha$-iron. Modelling and Simulation in Materials Science and Engineering, 4(5):473-492, 1996.

[21] W. A. Curtin. On lattice trapping of cracks. Journal of Materials Research, 5(07):15491560, 1990.

[22] G. E. Beltz, D. M. Lipkin, and L. L. Fischer. Role of crack blunting in ductile versus brittle response of crystalline materials. Physical Review Letters, 82(22):4468-4471, 1999.

[23] J. A. Zimmerman, C. L. Kelchner, P. A. Klein, J. C. Hamilton, and S. M. Foiles. Surface step effects on nanoindentation. Physical Review Letters, 87(16):165507, 2001. 
Table 1: Properties of 2D nearest-neighbor pair potential with tunable ductility, with $\alpha=$ 3.93, $r_{1}=1.05$, and $r_{2}=\sqrt{2}$. All critical energy release rates are normalized by $G_{I c}=2$. The value of $G_{I e}$ (Rice) is determined from the Rice criterion, and the corresponding "MS" values are determined from a quasi-static K/G-test in LAMMPS.

\begin{tabular}{|c|c|c|c|c|c|c|}
\hline Name & $-\varphi\left(r_{2}\right)$ & $r_{3}$ & $\gamma_{u s}(\mathrm{MS})$ & $\frac{G_{I e}(\text { Rice })}{G_{I c}}$ & $\frac{G_{I e}(\mathrm{MS})}{G_{I c}}$ & $\frac{G_{I c}(\mathrm{MS})}{G_{I c}}$ \\
\hline $\mathrm{A}, 2 \mathrm{D}$ & 0.378 & 1.706 & 0.157 & 0.56 & 0.62 & - \\
\hline $\mathrm{B}, 2 \mathrm{D}$ & 0.346 & 1.706 & 0.221 & 0.78 & 0.84 & - \\
\hline$C, 2 \mathrm{D}$ & 0.308 & 1.706 & 0.296 & 1.05 & - & 1.12 \\
\hline $\mathrm{D}, 2 \mathrm{D}$ & 0.264 & 1.706 & 0.384 & 1.37 & - & 1.09 \\
\hline $\mathrm{E}, 2 \mathrm{D}$ & 0.214 & 1.706 & 0.484 & 1.72 & - & 1.09 \\
\hline $\mathrm{F}, 2 \mathrm{D}$ & 0.160 & 1.700 & 0.591 & 2.10 & - & 1.15 \\
\hline $\mathrm{G}, 2 \mathrm{D}$ & 0.102 & 1.611 & 0.711 & 2.53 & - & 1.18 \\
\hline
\end{tabular}

Table 2: Properties of 3D nearest-neighbor pair potential with tunable ductility, with $\alpha=7.30, r_{1}=1.05$, and $r_{2}=\sqrt{3 / 2}$. All critical energy release rates are normalized by $G_{I c}=2 \sqrt{3}$. The value of $G_{I e}$ (Rice) is determined from the Rice criterion, and the corresponding "MS" values are determined from a quasi-static K/G-test in LAMMPS. Two crack orientations (in FCC) were explored: the (111) [110] system and the (110)[110] system. For the most ductile potential in the (111) [110] system, significant crack healing occurred before dislocation emission, so a blunted crack (created by removing a row of atoms) was used instead. For the most brittle potential in the (110) [110] system, the crack cleaved by kinking onto a (111) plane.

\begin{tabular}{|c|c|c|c|c|c|c|c|c|c|}
\hline \multirow[b]{2}{*}{ Name } & \multirow[b]{2}{*}{$-\varphi\left(r_{2}\right)$} & \multirow[b]{2}{*}{$r_{3}$} & \multirow[b]{2}{*}{$\gamma_{u s}(\mathrm{MS})$} & \multicolumn{3}{|c|}{ FCC, $(111)[\overline{1} 10]$} & \multicolumn{3}{|c|}{ FCC, $(110)[\overline{1} 10]$} \\
\hline & & & & $\frac{G_{I e}(\text { Rice })}{G_{I c}}$ & $\frac{G_{I l}(\mathrm{MS})}{G_{I c}}$ & $\frac{G_{I c}(\mathrm{MS})}{G_{I c}}$ & $\frac{G_{I e}(\text { Rice })}{G_{I c}}$ & $\frac{G_{I e}(\mathrm{MS})}{G_{I c}}$ & $\frac{G_{I c}(\mathrm{MS})}{G_{I c}}$ \\
\hline $\mathrm{A}, 3 \mathrm{D}$ & 0.318 & 1.391 & 0.356 & 0.53 & 0.88 (blunt) & - & 0.83 & 1.34 & - \\
\hline $\mathrm{B}, 3 \mathrm{D}$ & 0.266 & 1.391 & 0.473 & 0.71 & 0.98 & - & 1.11 & - & 1.27 \\
\hline$C, 3 D$ & 0.207 & 1.391 & 0.607 & 0.91 & 1.20 & - & 1.42 & - & 1.29 \\
\hline $\mathrm{D}, 3 \mathrm{D}$ & 0.143 & 1.360 & 0.759 & 1.13 & 1.46 & - & 1.77 & - & 1.39 \\
\hline$E, 3 D$ & 0.077 & 1.304 & 0.925 & 1.39 & 1.48 & - & 2.16 & - & 1.56 \\
\hline $\mathrm{F}, 3 \mathrm{D}$ & 0.009 & 1.247 & 1.111 & 1.66 & 1.78 & - & 2.59 & - & 1.75 (kinked) \\
\hline
\end{tabular}




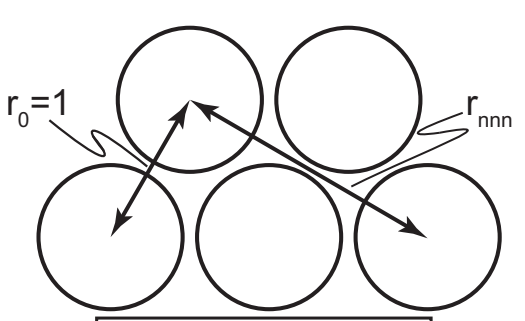

(a) Equilibrium

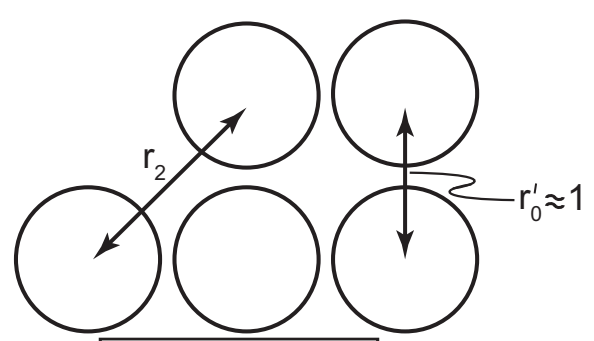

(b) Unstable

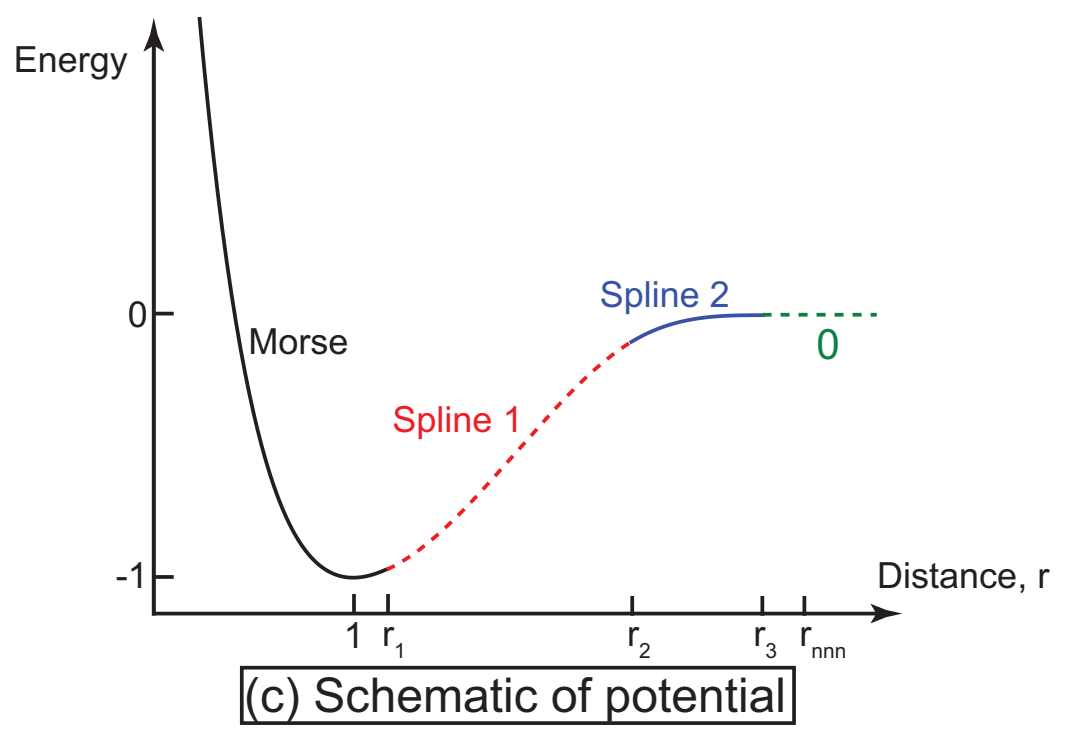

Figure 1: (a) Equilibrium configuration and (b) unstable configuration for dislocation nucleation in 2D hexagonal lattice. (c) Schematic of new nearest-neighbor pair potential. 


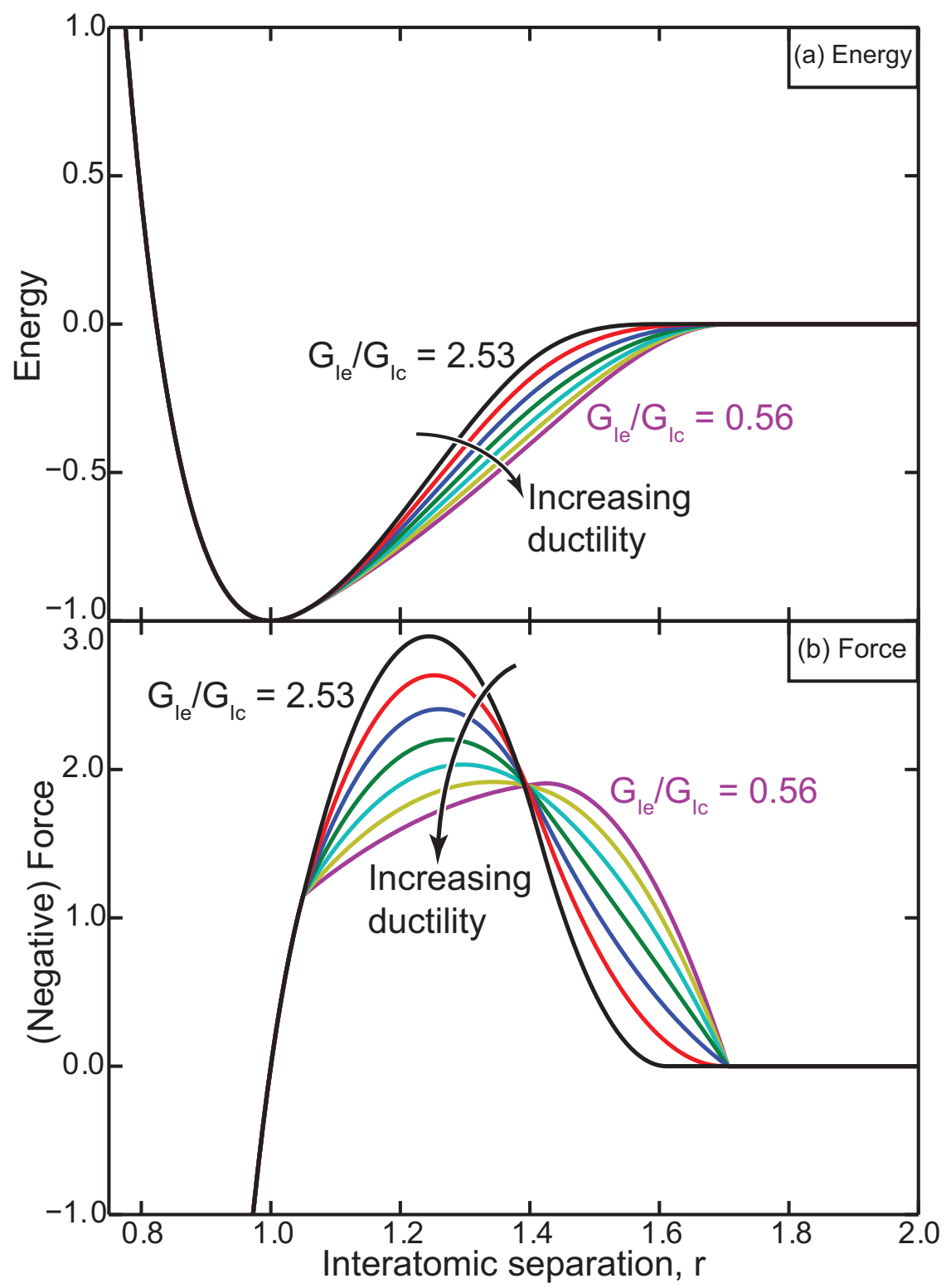

Figure 2: (a) Energy and (b) force vs. interatomic separation for new potential in 2D. The curves vary monotonically with intrinsic ductility: i.e., decreasing $G_{I e} / G_{I c}$. 


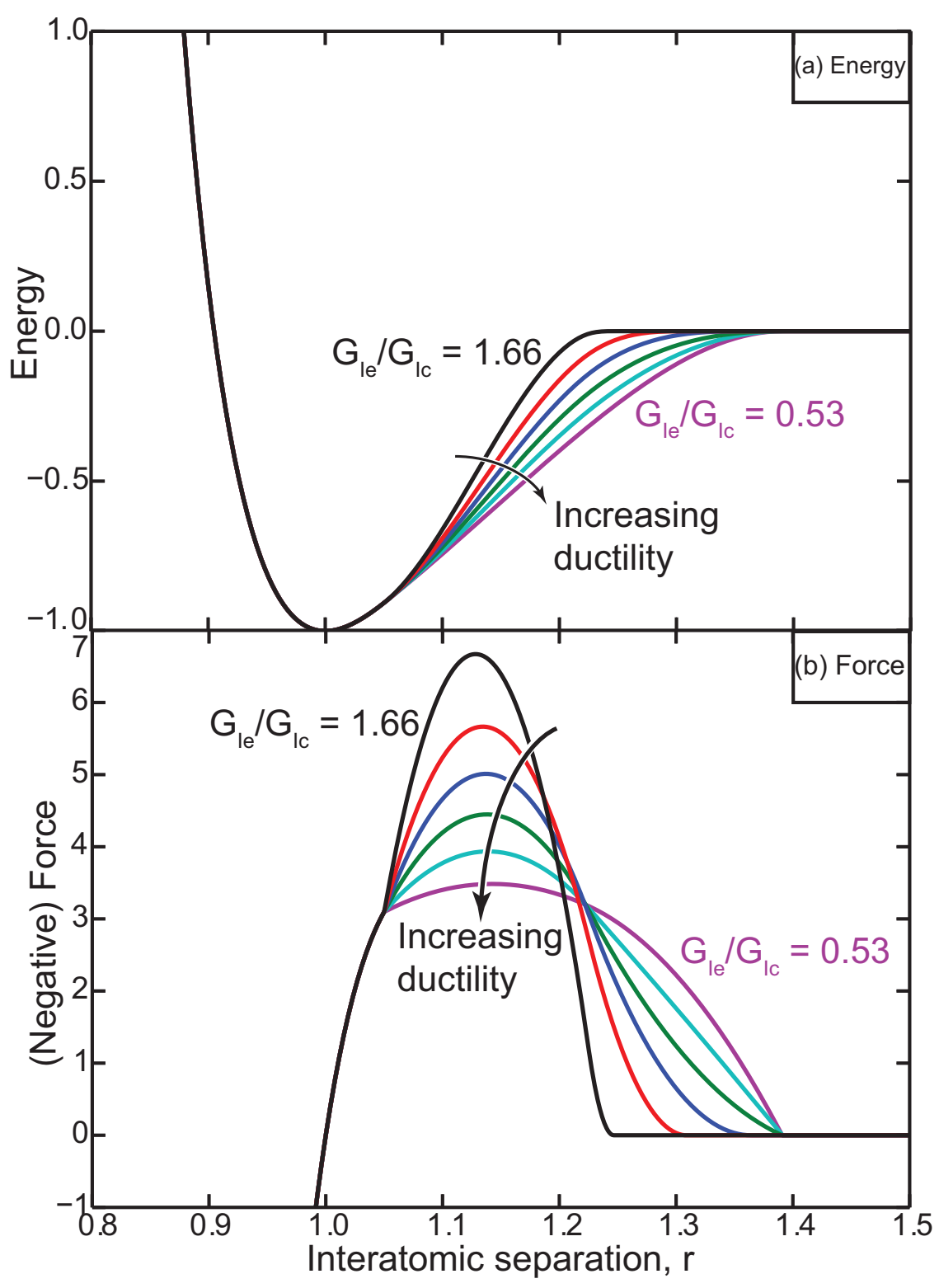

Figure 3: (a) Energy and (b) force vs. interatomic separation for new potential in 3D. The curves vary monotonically with intrinsic ductility: i.e., decreasing $G_{I e} / G_{I c}$. $G_{I e} / G_{I c}$ ratios are for FCC, with (111) crack plane and [110] crack front. 

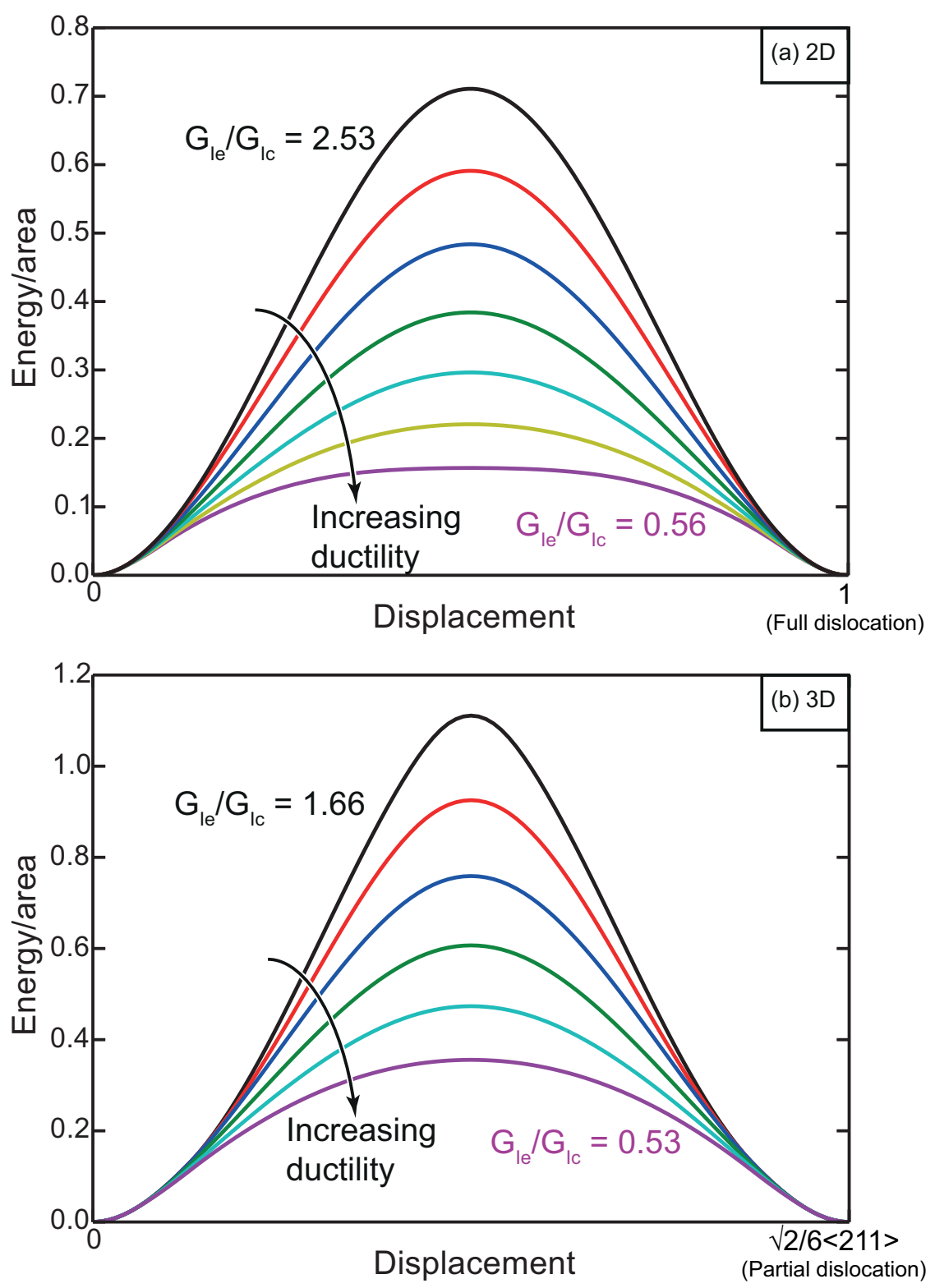

Figure 4: Generalized stacking fault energy curves in (a) 2D and (b) 3D. The curves vary monotonically with intrinsic ductility: i.e., decreasing $G_{I e} / G_{I c}$. $G_{I e} / G_{I c}$ ratios in 3D are for FCC, with (111) crack plane and [110] crack front. 
Table 3: Spline constants for the 2D nearest-neighbor pair potential with tunable ductility, with $\alpha=3.93, r_{1}=1.05$, and $r_{2}=\sqrt{2}$.

\begin{tabular}{ccccccccccc}
\hline Name & $-\varphi\left(r_{2}\right)$ & $r_{3}$ & $A_{1}$ & $B_{1}$ & $C_{1}$ & $D_{1}$ & $A_{2}$ & $B_{2}$ & $C_{2}$ & $D_{2}$ \\
\hline A, 2D & 0.378 & 1.706 & -1.402 & 6.214 & -7.262 & 1.428 & -8.058 & 34.453 & -47.198 & 20.255 \\
B, 2D & 0.346 & 1.706 & -2.973 & 11.975 & -14.165 & 4.144 & -5.867 & 24.257 & -31.535 & 12.332 \\
C, 2D & 0.308 & 1.706 & -4.838 & 18.817 & -22.364 & 7.368 & -3.266 & 12.150 & -12.934 & 2.923 \\
D, 2D & 0.264 & 1.706 & -6.998 & 26.740 & -31.856 & 11.101 & -0.255 & -1.870 & 8.604 & -7.972 \\
E, 2D & 0.214 & 1.706 & -9.453 & 35.742 & -42.644 & 15.344 & 3.168 & -17.801 & 33.079 & -20.352 \\
F, 2D & 0.160 & 1.700 & -12.105 & 45.471 & -54.301 & 19.929 & 6.871 & -35.037 & 59.556 & -33.744 \\
G, 2D & 0.102 & 1.611 & -15.461 & 57.703 & -68.886 & 25.643 & 13.364 & -64.594 & 104.067 & -55.887 \\
\hline
\end{tabular}

Table 4: Parameters for the 3D nearest-neighbor pair potential with tunable ductility, with $\alpha=7.30, r_{1}=1.05$, and $r_{2}=\sqrt{3 / 2}$.

\begin{tabular}{ccccccccccc}
\hline Name & $-\varphi\left(r_{2}\right)$ & $r_{3}$ & $A_{1}$ & $B_{1}$ & $C_{1}$ & $D_{1}$ & $A_{2}$ & $B_{2}$ & $C_{2}$ & $D_{2}$ \\
\hline A, 3D & 0.318 & 1.391 & -15.047 & 51.581 & -55.454 & 17.871 & -23.550 & 82.823 & -93.717 & 33.492 \\
B, 3D & 0.266 & 1.391 & -35.301 & 120.620 & -133.449 & 47.096 & -1.691 & -2.867 & 17.791 & -14.648 \\
C, 3D & 0.207 & 1.391 & -58.280 & 198.953 & -221.944 & 80.255 & 23.110 & -100.092 & 144.310 & -69.268 \\
D, 3D & 0.143 & 1.360 & -84.000 & 286.563 & -320.854 & 117.296 & 52.780 & -215.999 & 294.656 & -133.985 \\
E, 3D & 0.077 & 1.304 & -119.853 & 407.925 & -457.132 & 168.089 & 128.108 & -503.143 & 658.693 & -287.444 \\
F, 3D & 0.009 & 1.247 & -194.610 & 658.701 & -736.502 & 271.488 & 857.883 & -3208.407 & 3999.719 & -1662.066 \\
\hline
\end{tabular}

